\title{
Thermoresponsive Poly(2-oxazoline) Block Copolymers Exhibiting Two Cloud Points: Complex Multistep Assembly Behavior
}

\author{
Loan T. T. Trinh, ${ }^{\dagger}$ Hanneke M. L. Lambermont-Thijs, ${ }^{\ddagger}$ Ulrich S. Schubert, ${ }^{\ddagger}$, Richard Hoogenboom, $^{*, \|}$

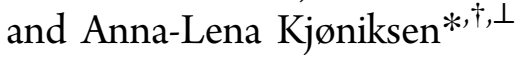 \\ ${ }^{\dagger}$ Department of Chemistry, University of Oslo, P.O. Box 1033, Blindern, 0315 Oslo, Norway \\ ${ }^{\ddagger}$ Laboratory of Macromolecular Chemistry and Nanoscience, Eindhoven University of Technology, Den Dolech 2, 5612AZ \\ Eindhoven, The Netherlands \\ ${ }^{\S}$ Laboratory of Organic and Macromolecular Chemistry (IOMC) and Jena Center for Soft Matter (JCSM), \\ Friedrich-Schiller-University Jena, Humboldtstr. 10, 07743 Jena, Germany \\ "Supramolecular Chemistry Group, Department of Organic Chemistry, Ghent University, Krijgslaan 281 S4, B-9000 Ghent, Belgium \\ ${ }^{\perp}$ Department of Pharmacy, School of Pharmacy, University of Oslo, P.O. Box 1068, Blindern, 0316 Oslo, Norway
}

ABSTRACT: Aqueous solutions of poly(2-oxazoline) block copolymers consisting of a 2-ethyl-2-oxazoline block and a block consisting of a random copolymer of 2-ethyl-2-oxazoline and 2-n-propyl-2-oxazoline (PEtOx-block-P(EtOx-stat-Pro$\mathrm{pOx})$ ) have been studied by dynamic light scattering (DLS), static light scattering (SLS), and turbidimetry. Even at temperatures significantly below the lower critical solution
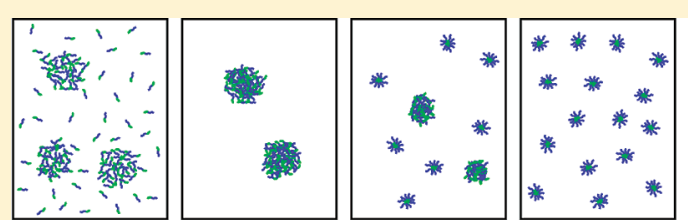

Increasing Temperature temperature (LCST), polymer unimers are found to coexist with a few large aggregates with an open structure. When heated, the systems exhibit an intricate transmittance behavior whereby the samples becomes visually clear again after an initial cloud point and then exhibit a second cloud point at even higher temperatures. The DLS data indicate that the aggregates formed around the first cloud point restructure and fragment into smaller micelle-like structures ascribed to further dehydration of the more hydrophobic PPropOx containing block, causing the samples to become optically clear again. The observed fragmentation is confirmed by the SLS experiments. At even higher temperatures, both blocks become hydrophobic, causing the formation of large, compact aggregates, resulting in a second cloud point.

\section{INTRODUCTION}

Thermoresponsive polymers with a lower critical solution temperature (LCST) are interesting for various applications such as controlled drug delivery, ${ }^{1-4}$ enhanced oil recovery, ${ }^{5}$ sensors, ${ }^{6-8}$ water purification, ${ }^{5,9}$ and in vivo imaging for, e.g., tumor diagnosis. ${ }^{10}$ Solutions and suspensions of polymers with a LCST behavior are characterized by a transition from clear to turbid solutions when heated, and this transition temperature is denoted the cloud point temperature of the solution. The temperature-induced phase transition is an entropically driven process, which is based on the increased mobility of the water molecules that are released into the bulk water upon dehydration. The cloud point temperature generally depends on various factors such as polymer concentration, ${ }^{11-15}$ molecular weight of the polymer, ${ }^{12-14,16}$ grafting $^{17}$ or branching $^{18}$ of the polymer, copolymerization with other monomers, $2,12,13,16,19-23$ the architecture of the resulting copolymers, ${ }^{19}$ and the addition of cosolvents ${ }^{24,25}$ or cosolutes such as salts ${ }^{11,26}$ or surfactants. ${ }^{11,26}$ The decreased transmittance of the samples is due to the self-assembly of the partially dehydrated polymers into large, compact aggregates. For some, mostly block copolymer, systems, a continued heating after the cloud point causes the samples to become more transparent again over a limited temperature range before a second transition into more turbid samples occur at even higher temperatures. ${ }^{15,27-30}$ Both cloud points indicate the formation of large aggregates, while the reappearance of a more transparent region at intermediate temperatures has been contributed to the formation of compact micelles that are too small to scatter the light. ${ }^{27}$ On the basis of theoretical considerations, ${ }^{31,32}$ it can be shown that a temperature-induced increase of the transmittance values can be due to swelling of the polymer entities, fragmentation into smaller structures, or the formation of very large and compact structures which bring the samples into a range where the Mie theory predicts an oscillation of the transmittance values as the aggregates becomes even larger and/or more compact. In order to reach the latter stage, the aggregates need to be both large (typically at least $1 \mu \mathrm{m}$ ) and very compact at the same time.

One of the most popular thermoresponsive polymers is poly(N-isopropylacrylamide) (PNIPAAM), which has a LCST of about $32{ }^{\circ} \mathrm{C}$. Even though the cloud point of PNIPAAM can

Received: March 20, 2012

Revised: $\quad$ May 2, 2012

Published: May 11, 2012 
be tuned by copolymerization with various comonomers, ${ }^{2,20-22}$ other thermoresponsive polymers exhibiting LCST behavior can offer interesting alternatives. A class of polymers with promising properties is poly(2-oxazoline) $\mathrm{s}^{33,34}$ which have cloud points that are easily tunable over a wide range of temperatures. $^{13,35,36}$ These polymers have the additional advantage of exhibiting low toxicity, ${ }^{37,38}$ being biocompatible, ${ }^{39-41}$ and provide stealth behavior similar to poly(ethylene glycol). ${ }^{39,40}$ They have also been found to have a very high solubilization capacity for highly water insoluble drugs. ${ }^{3}$

In this contribution, we describe the temperature-induced changes in aqueous solutions of copolymers of 2-ethyl-2oxazoline (EtOx) and 2- $n$-propyl-2-oxazoline (PropOx). The copolymers consist of a PEtOx block and a block that is a random copolymer of EtOx and PropOx, denoted $\mathrm{PEtOx}_{80^{-}}$ block-P $\left(\mathrm{EtOx}_{x}\right.$-stat-PropOx $\left.\mathrm{x}_{40-x}\right)$. Three different copolymers with a high amount of PropOx in the second block $(x=0,4$, or 8) have been studied. PEtOx has a cloud point which is significantly higher than what is observed for PPropOx. ${ }^{13}$ Accordingly, at low temperatures the entire polymer is relatively hydrophilic. When the temperature is raised to intermediate temperatures, the block containing mostly PropOx partially dehydrates and becomes more hydrophobic while the EtOx block is still quite hydrophilic. At higher temperatures, both blocks are partially dehydrated and, thus, hydrophobic. Consequently, when the samples are heated, the polymers exhibit a gradual transition from hydrophilic to amphiphilic to hydrophobic. In this contribution we will employ dynamic light scattering (DLS) in combination with turbidity data to scrutinize the intricate temperature-induced transformations and the aggregation behavior of these copolymers. In addition, static light scattering (SLS) has been conducted on one of the samples, confirming the results found by the DLS and turbidity experiments.

\section{MATERIALS AND METHODS}

Materials. Block copolymers consisting of a first block of 2-ethyl-2oxazoline and a second block consisting of a random copolymer of 2ethyl-2-oxazoline and 2-n-propyl-2-oxazoline denoted $\mathrm{PEtO}_{80}$-block-

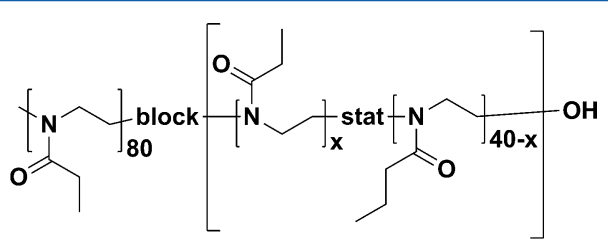

Figure 1. Schematic representation of the chemical structure of the $\mathrm{PEtOx}_{80}$-block-P(EtOx $x_{x}$-stat-PropOx $\left.{ }_{40-x}\right)$ copolymers. The amount of EtOx in the second block is: $x=0,4$, or 8 .

$\mathrm{P}\left(\mathrm{EtOx}_{x}\right.$-stat-PropOx $\left.\mathrm{x}_{40-x}\right)(x=0,4$ or 8$)$ (see Figure 1$)$ have been synthesized by a sequential monomer addition procedure following our previously reported methodologies. ${ }^{13,42}$ After the polymerization, the polymers were isolated by drying in a vacuum oven. ${ }^{1} \mathrm{H}$ NMR spectroscopy (Varian Mercury $400 \mathrm{MHz}$ spectrometer in $\mathrm{CDCl}_{3}$; the residual protonated solvent signals were used as reference) confirmed the absence of trace amounts of solvents and residual monomers. Size exclusion chromatography (SEC) was performed on a Shimadzu system with a SCL-10A system controller, a LC-10AD pump, a RID$10 \mathrm{~A}$ refractive index detector, and PSS gram 30 (pore size 30 Á; bead size $10 \mu \mathrm{m} ; 100-10000 \mathrm{Da}$ ) and PSS gram 1000 (pore size $1000 \AA^{\prime}$; bead size $10 \mu \mathrm{m} ; 1000-1000000 \mathrm{Da})$ columns in series at $60{ }^{\circ} \mathrm{C}$. A solution of $N, N$ dimethylacetamide (DMAc) containing $2.1 \mathrm{~g} \mathrm{LiCl} / \mathrm{L}$ was used as an eluent at a flow rate of $1 \mathrm{~mL} / \mathrm{min}$. The average molecular weights were calculated against poly(styrene) (PS) calibration standards.

PEtOx 80 -block-PPropO $x_{40}$. SEC PEtOx first block: $M_{\mathrm{n}}=6800 \mathrm{~g} /$ mol, PDI $=1.10$; SEC block copolymer: $M_{\mathrm{n}}=8700 \mathrm{~g} / \mathrm{mol}$, PDI $=$ 1.22. ${ }^{1} \mathrm{H}$ NMR: $\mathrm{DP}_{\mathrm{EtOx}}=78, \mathrm{DP}_{\text {block copolymer }}=114$, PropOx content second block: $>96 \%$.

PEtOx ${ }_{80}$-block-P(EtOx $x_{4}$ stat-PropOx $\left.{ }_{36}\right)$. SEC PEtOx first block: $M_{\mathrm{n}}$ $=6500 \mathrm{~g} / \mathrm{mol}$, PDI $=1.09$; SEC block copolymer: $M_{\mathrm{n}}=8200 \mathrm{~g} / \mathrm{mol}$, $\mathrm{PDI}=1.23 .{ }^{1} \mathrm{H}$ NMR: $\mathrm{DP}_{\mathrm{EtOx}}=79, \mathrm{DP}_{\text {block copolymer }}=113$, PropOx content second block: $\sim 91 \%$.

PEtOx $x_{80}$-block-P(EtOx $x_{8}$-stat-PropOx $\left.{ }_{32}\right)$. SEC PEtOx first block: $M_{n}$ $=6600 \mathrm{~g} / \mathrm{mol}$, PDI $=1.09$; SEC block copolymer: $M_{\mathrm{n}}=9200 \mathrm{~g} / \mathrm{mol}$, $\mathrm{PDI}=1.20 .{ }^{1} \mathrm{H}$ NMR: $\mathrm{DP}_{\mathrm{EtOx}}=79, \mathrm{DP}_{\text {block copolymer }}=119$, PropOx content second block: $\sim 79 \%$.

Transmittance Measurements. The transmittance of the polymer solutions $(5 \mathrm{mg} / \mathrm{mL})$ were determined by turbidity measurements using a Crystal 16 (Avantium Technologies, The Netherlands) connected to a Julabo FP40 cryostat. Turbidity of the solutions was measured by the transmission of red light through the sample vial as a function of the temperature while stirring. Solutions of the polymers were prepared in deionized water (Laborpure, Behr Labor Technik) and were stirred at room temperature until all polymer was dissolved or dispersed. The samples were measured from 20 to $80{ }^{\circ} \mathrm{C}$ with a heating rate of $1{ }^{\circ} \mathrm{C} / \mathrm{min}$.

Dynamic Light Scattering. The dynamic light scattering (DLS) experiments were carried out by an ALV/CGS-8F goniometer system, with 8 fiber-optical detection units, from ALV-GmbH, Langen, Germany. The polymer solutions $(5 \mathrm{mg} / \mathrm{mL})$ were filtered in an atmosphere of filtered air through a $5 \mu \mathrm{m}$ filter (Millipore) directly into precleaned $10 \mathrm{~mm}$ NMR tubes (Wilmad Glass Co.).

Assuming that the scattering of the incoming light exhibit Gaussian statistics, the experimentally recorded intensity autocorrelation function $g^{2}(q, t)$ is directly linked to the theoretically amenable firstorder electric field autocorrelation function $g^{1}(q, t)$ through the Siegert ${ }^{43}$ relationship $g^{2}(q, t)=1+B\left|g^{1}(q, t)\right|^{2}$, where $B(\leq 1)$ represents an instrumental parameter, and the magnitude of the wave vector, $q$, is $q=(4 \pi n / \lambda) \sin (\theta / 2), \lambda$ is the wavelength of the incident light in a vacuum, $\theta$ is the scattering angle, and $n$ is the refractive index of the medium.

Depending on the conditions, the correlation functions were found to exhibit either one or two relaxation modes. The correlation functions with two relaxation modes were fitted by the sum of a single and a stretched exponential:

$$
g^{1}(q, t)=A_{\mathrm{f}} \exp \left(-t / \tau_{\mathrm{f}}\right)+A_{\mathrm{s}} \exp \left[-\left(t / \tau_{\mathrm{se}}\right)^{\beta}\right]
$$

where $A_{\mathrm{f}}+A_{\mathrm{s}}=1$. The parameters $A_{\mathrm{f}}$ and $A_{\mathrm{s}}$ are the amplitudes and $\tau_{\mathrm{f}}$ and $\tau_{\text {se }}$ are the relaxation times for the fast and the slow relaxation modes, respectively. The parameter $\tau_{\mathrm{se}}$ is an effective relaxation time, and the stretched exponent $\beta(0<\beta \leq 1)$ is a measure of the width of the distribution of the relaxation times. The mean slow relaxation time is given by $\tau_{\mathrm{s}}=\left(\tau_{\mathrm{se}} / \beta\right) \Gamma(1 / \beta)$, where $\Gamma$ is the gamma function. We also tried fitting the correlation functions using the sum of two stretched exponentials. However, this always resulted in a stretched exponent of the fast mode which was very close to one, and therefore a single exponential was used in order to reduce the number of fitting parameters. The single-exponential nature of the fast mode shows that it probes entities with a narrow size distribution.

The correlation functions where only one mode was evident were fitted by

$$
g^{1}(q, t)=\exp \left[-\left(t / \tau_{\mathrm{se}}\right)^{\beta}\right]
$$

where the symbols have the same meaning as for eq 1 .

In order to determine the number of relaxation modes for each correlation function, residual plots have been employed. If a fit to eq 1 gives much better residuals than eq 2 , as shown for the system in Figure 2a, the correlation functions have been deemed to contain two relaxation modes. The correlation functions were fitted using one mode when they exhibited similar residual plots from eqs 1 and 2, like 


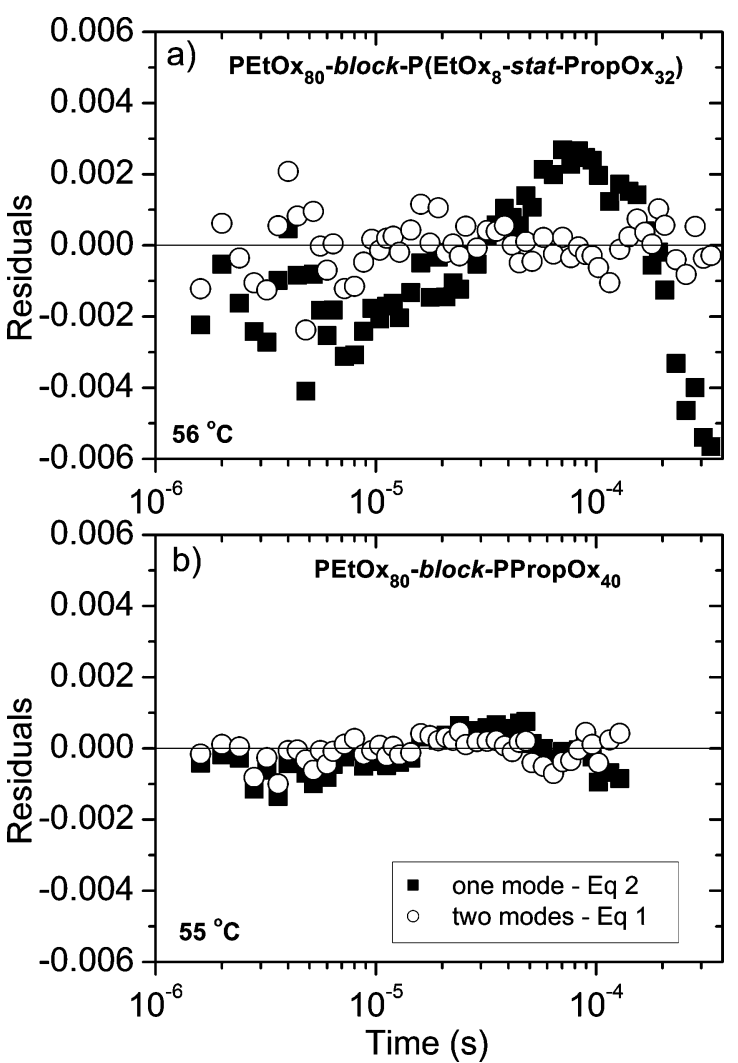

Figure 2. Residual plots (differences between the fitted and measured data) for some representative systems fitted with both eq 1 (two modes) and eq 2 (one mode). (a) For a system that exhibits two relaxation modes and (b) for a system that exhibits only one relaxation mode.

the system in Figure $2 \mathrm{~b}$. At these conditions, a fitting using eq 1 also resulted in effects such as $\tau_{\mathrm{f}}$ and $\tau_{\mathrm{s}}$ values very close to each other, very low amplitudes for one of the relaxation modes, and/or large errors in the fitting parameters.

The $q$-dependency of the fast and slow relaxation times can be quantified by $\tau_{\mathrm{f}}^{-1} \sim q^{\alpha_{\mathrm{f}}}$ and $\tau_{\mathrm{s}}^{-1} \sim q^{\alpha_{\mathrm{s}}}$. It was found that $\alpha_{\mathrm{f}} \approx 2$ at all conditions. This shows that this relaxation mode is diffusive, and the Stokes-Einstein relationship can be used to calculate the hydrodynamic radius of the fast relaxation mode: $R_{\mathrm{h}, \mathrm{f}}=\left(k_{\mathrm{B}} T\right) /\left(6 \pi \eta D_{\mathrm{f}}\right)$, where $k_{\mathrm{B}}$ is the Boltzmann constant, $T$ is the absolute temperature, and $\eta$ is solvent viscosity. The mutual diffusion coefficient of the fast mode can be expressed as $D_{\mathrm{f}}=1 /\left(\tau_{\mathrm{f}} q^{2}\right)$. The values of $\alpha_{\mathrm{s}}$ were found to be higher than 2 at some temperatures (see Figure 3). A q-dependency higher than 2 might be caused by interactions between the particles ${ }^{44,45}$ or if $q R \gg 1$ by the influence of internal motions within the particles. ${ }^{46}$ The Stokes-Einstein relationship is not valid when $\alpha_{\mathrm{s}}$ $>2$, and using it under these conditions will result in different sizes depending on the scattering angle employed in the measurements. However, even though the absolute values will not be correct, the trends in the data should indicate whether the sizes are increasing or decreasing. We have therefore chosen to represent the values calculated from the slow relaxation mode as an apparent hydrodynamic radius, $R_{\mathrm{h}, s}$, using a scattering angle of $107^{\circ}: R_{\mathrm{h}, \mathrm{s}}=\left(k_{\mathrm{B}} T\right) /$ $\left(6 \pi \eta D_{\mathrm{s}}\right)$, where the mutual diffusion coefficient of the slow mode is $D_{\mathrm{s}}$ $=1 /\left(\tau_{s} q^{2}\right)$. The amplitudes of the two relaxation modes are angle dependent (the slow mode becomes more dominant at low angles). The scattering angle of $107^{\circ}$ was chosen since it was found to give the best resolution of both of the two modes.

Static Light Scattering. The static light scattering (SLS) experiments were carried out by an ALV/CGS-8F goniometer system, with 8 fiber-optical detection units, from ALV-GmbH, Langen, Germany. The polymer solutions $(5 \mathrm{mg} / \mathrm{mL})$ were filtered in an

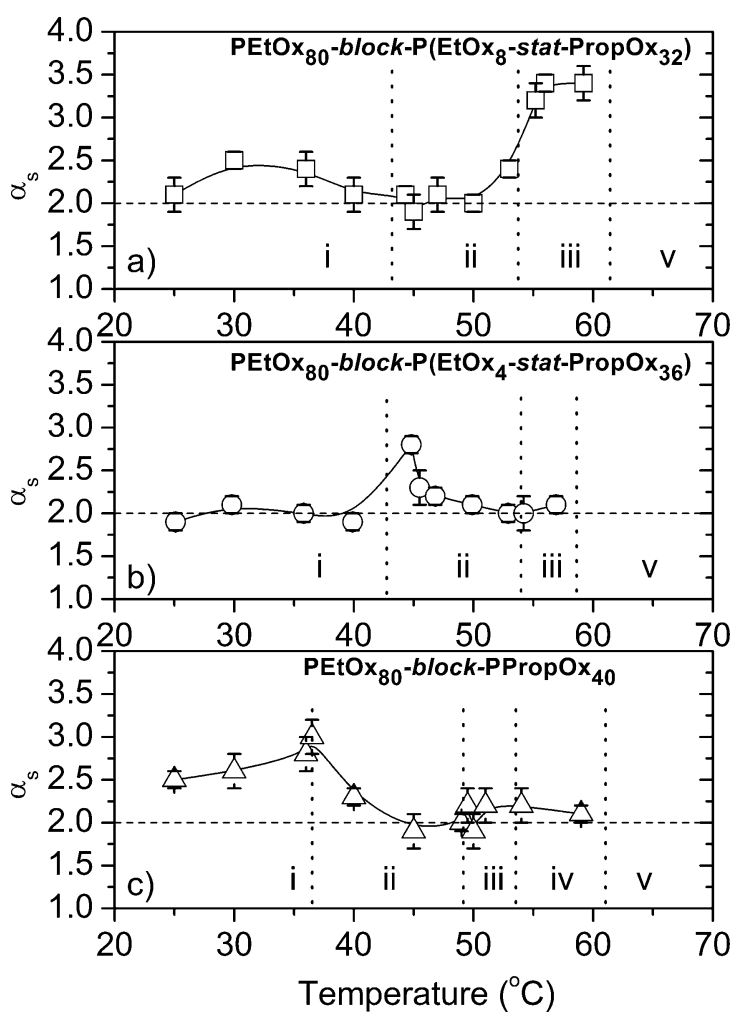

Figure 3. The $q$-dependency of the slow relaxation mode of the indicated systems at a polymer concentration of $5 \mathrm{mg} / \mathrm{mL}$.

atmosphere of filtered air through a $5 \mu \mathrm{m}$ filter (Millipore) directly into precleaned $10 \mathrm{~mm}$ NMR tubes (Wilmad Glass Co.). The heating rate of $1{ }^{\circ} \mathrm{C} / \mathrm{min}$ used for the transmittance measurements and the DLS experiments could not be used for the SLS experiments, since the temperature of the sample would significantly change during the time it takes to perform a measurement. The SLS experiments were therefore conducted at selected fixed temperatures. The samples were heated from $25{ }^{\circ} \mathrm{C}$ to the considered temperature with a heating rate of $1^{\circ} \mathrm{C} / \mathrm{min}$ and then measured after the sample had stabilized at the considered temperature. The standard (toluene) and solvent (water) used to calculate the Rayleigh ratio, $R_{\mathrm{vv}}(q)$, were also measured at each of the considered temperatures. Since the aggregation process is expected to be concentration dependent, the radius of gyration, $R_{g}$, and the weight-average molecular weight of the aggregates, $M_{w}$, could not be measured using a concentration series. We have therefore made the approximation that the relatively low concentration $(c)$ of $5 \mathrm{mg}$ / $\mathrm{mL}$ is in the region where $c \rightarrow 0$. In the low- $q$ region, this approximation yields 47,48

$$
\frac{K c}{R_{\mathrm{vv}}(q)} \approx \frac{1}{M_{\mathrm{w}}}\left(1+\frac{1}{3} R_{\mathrm{g}}{ }^{2} q^{2}\right)
$$

where $K=\left(4 \pi^{2} n^{2}\right) /\left(N_{\mathrm{A}} \lambda^{4}\right)(\mathrm{d} n / \mathrm{d} c)^{2}, N_{\mathrm{A}}$ is Avogadro's constant, and $\mathrm{d} n / \mathrm{d} c$ is the refractive index increment.

\section{RESULTS AND DISCUSSION}

For PEtOx and PPropOx homopolymers, a single transition from clear to turbid samples have been reported. ${ }^{13,35}$ Interestingly, $\mathrm{PEtOx}_{80}$-block-P(EtOx $x_{x}$-stat-PropOx $\left.\mathrm{x}_{40-x}\right)$ with $x$ $=0,4$, or 8 revealed an initial drop in transmittance upon heating followed by an unexpected increase in the transmittance data upon further heating (see Figure 4). The minimum in the transmittance curves becomes deeper as the amount of PropOx in the copolymers is raised. At even higher temperatures, the transmittance decreases again resulting in a second cloud point transition. Visual inspection of the 


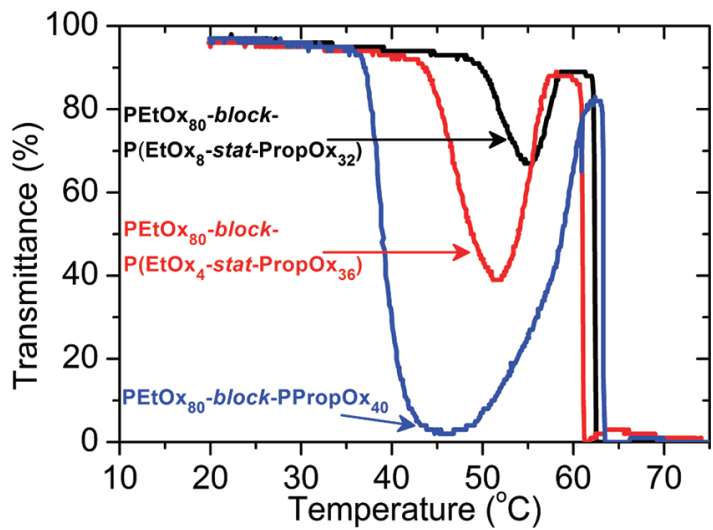

Figure 4. Transmittance versus temperature plots of the block copolymers at a polymer concentration of $5 \mathrm{mg} / \mathrm{mL}$.

copolymer solutions revealed that they are visually clear at low temperatures. $\mathrm{PEtO}_{80}$-block-P( $\left(\mathrm{EtOx}_{4}\right.$-stat-PropO $\left.\mathrm{x}_{36}\right)$ and $\mathrm{PEtOx}_{80}$-block-PPropOx $\mathrm{x}_{40}$ have a cloudy appearance around the minimum in the transmittance values and then become visually clear again at higher temperatures. The smaller drop in transmittance observed for $\mathrm{PEtO}_{80}-$ block-P(EtOx $\mathrm{x}_{8}$-stat-Pro$\left.\mathrm{pOx}_{32}\right)$ is not very obvious to the naked eye. At high temperatures all three samples look completely opaque. No precipitation was observed in the considered temperature range using a heating rate of $1{ }^{\circ} \mathrm{C} / \mathrm{min}$. In order to gain a better understanding of what causes these particular transmittance versus temperature profiles, dynamic light scattering measurements were conducted on the copolymer solutions.

Figure 5 show the measured correlation functions obtained by DLS at a scattering angle of $107^{\circ}$. The data have been normalized to compensate for the temperature dependency of the solvent viscosity. At low temperatures, the correlation functions clearly exhibit two relaxation modes. As the temperature is increased, only one relaxation mode is observed in the correlation functions. At even higher temperatures there is a reappearance of two modes, and at very high temperatures, only one mode is evident again. It is also clear from Figure 5 that the relaxation times are changing in a very complex manner when the temperature is increased; i.e., there is not a logical continuous shift from fast to slow relaxation upon heating. In order to quantify these effects, the correlation functions have been fitted with eq 1 or eq 2 depending on the number of relaxation modes observed.

In order to interpret the results from the DLS measurements, it is important to understand the various effects that can affect the sizes of associative polymer systems. The comparison with the transmittance data can be of great help in the interpretation of the data, too. Basically, the transmittance of a dilute polymer sample is dependent on the polymer concentration and on the size and compactness of the polymer entities in the sample. ${ }^{31,32}$ By using the equations described in ref 32 , it can be found that unless the polymer aggregates are both very large (typically $R_{\mathrm{h}}$ $>1 \mu \mathrm{m})$ and very compact at the same time, the changes in size and transmittance are caused by a limited number of different processes as summarized in Table 1. Generally, larger objects with similar refractive index will scatter more light; thus, aggregation leads to a decrease in transmittance and fragmentation will increase the transmittance. In contrast, swelling results in larger objects that have a refractive index closer to the medium yielding higher transmittance. Similarly, shrinking due to dehydration leads to a higher difference in

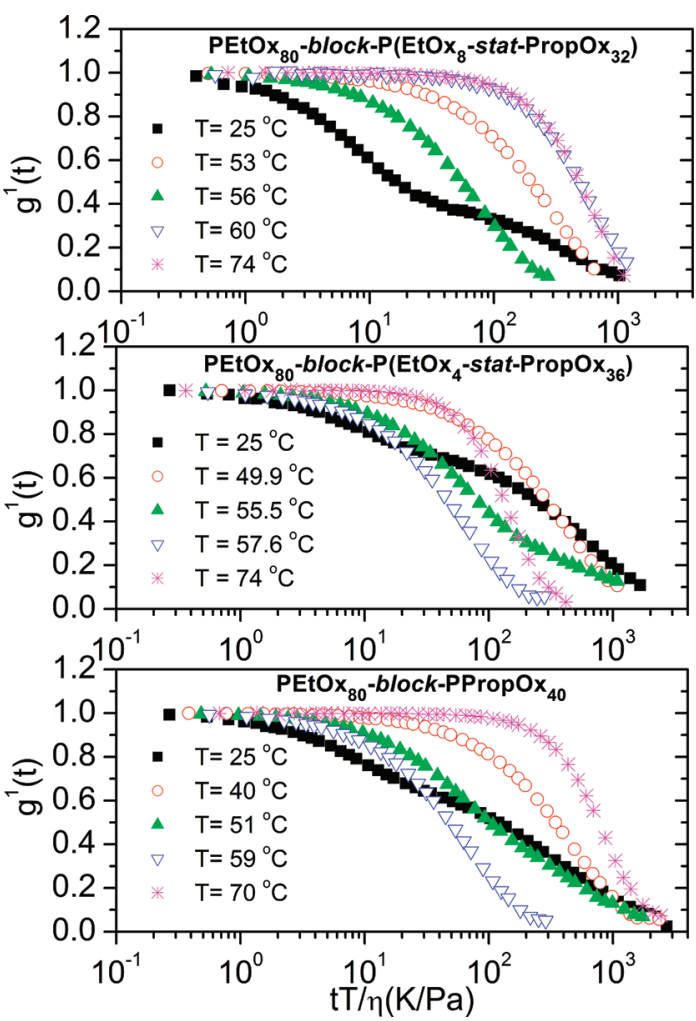

Figure 5. First-order electric field correlation functions (at a scattering angle of $107^{\circ}$; every second data point is shown) versus the quantity $t T / \eta$ (trivial changes of the solvent viscosity with temperature are thereby accounted for) for the indicated systems at a polymer concentration of $5 \mathrm{mg} / \mathrm{mL}$.

Table 1. Overview over How Different Processes Will Affect the Size and Transmittance Data of a Dilute Polymer Sample at a Constant Polymer Concentration, Provided the Polymer Entities Are Not Both Very Large and Very Compact at the Same Time

$\begin{array}{lll}\text { aggregation } & \text { decreasing transmittance, } \downarrow & \text { increasing size, } R_{\mathrm{h}} \uparrow \\ \text { swelling } & \text { increasing transmittance, } \uparrow & \text { increasing size, } R_{\mathrm{h}} \uparrow \\ \text { fragmentation } & \text { increasing transmittance, } \uparrow & \text { decreasing size, } R_{\mathrm{h}} \downarrow \\ \text { shrinking } & \text { decreasing transmittance, } \downarrow & \text { decreasing size, } R_{\mathrm{h}} \downarrow\end{array}$

refractive index between the objects and the solvent giving rise to stronger scattering and, thus, lower transmittance. However, it is of course important to remember that the forces that causes aggregation, e.g., enhanced hydrophobicity due to partial dehydration, will often also be accompanied by shrinking of the same aggregates. Therefore, these two effects often occur simultaneously. ${ }^{20,48-51}$ Even though both effects will give rise to lower transmittance values, the overall size change will depend upon whether the size increase from the aggregation process or the size decrease from shrinking is most dominant.

It is also important to keep in mind that thermosensitive polymers are sometimes found to exhibit associative interactions even at temperatures significantly below their lower critical solution temperature. ${ }^{22,52,53}$ In addition, a temperature increase will render the polymers more hydrophobic over a wide range of temperatures both before and after the cloud point temperature is reached..$^{30,48,49,52}$ Keeping these general considerations in mind, we will now examine the size changes in our poly(2-oxazoline) systems. 
The apparent hydrodynamic radii of the three copolymers are plotted together in Figure 6, illustrating how the radii are

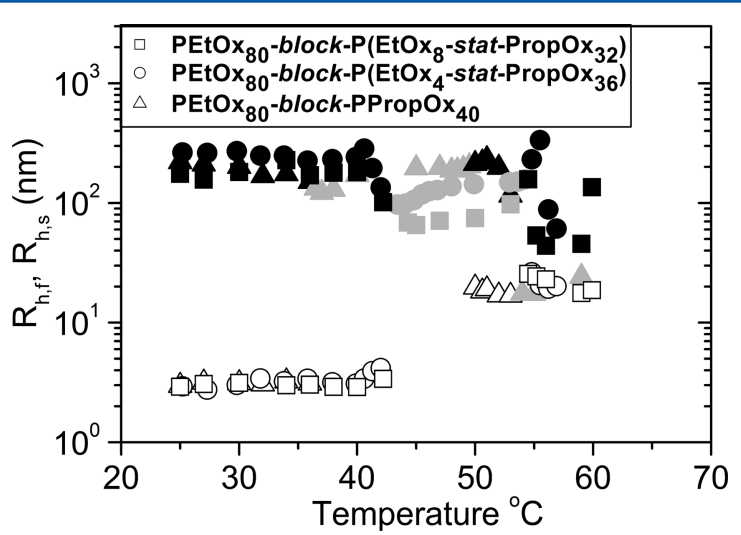

Figure 6. Apparent hydrodynamic radius of the indicated systems as a function of temperature at a polymer concentration of $5 \mathrm{mg} / \mathrm{mL}$. Open symbols represent sizes calculated from the fast relaxation mode, closed black symbols represent sizes calculated from the slow relaxation mode, and closed gray symbols represent sizes calculated from correlation functions that exhibit only one relaxation mode.

affected by the amount of PropOx in the second block. For clarity, each of the polymer systems is also displayed separately in Figure 7 together with the corresponding transmittance data. From the DLS data, the behavior of these copolymer systems can be divided into five different temperature regions:

Region i. In this region, which occurs at low temperatures, the transmittance of the samples is high and decreases slightly with temperature (see Figure 7). As can be seen from Figure 7, the correlation functions display two relaxation modes. The first mode (open symbols) gives a hydrodynamic radius of about 3 $\mathrm{nm}$, independent of the amount of PropOx in the copolymer (see Figure 6), suggesting that we are probing the copolymer unimers, i.e., single polymer chains. These have a constant size since the different copolymers have approximately the same molecular weight and a fairly similar composition. When the temperature is increased, the size of the unimers remains constant. As region ii is approached, there is a slight increase in sizes indicating an incipient aggregation of the unimers due to the temperature-induced enhanced hydrophobicity of the samples.

The existence of a second mode with a much longer relaxation time indicates that the unimers are coexisting with large aggregates. This illustrates that the copolymers exhibit a somewhat hydrophobic nature even at these low temperatures. The sizes of the aggregates are in the range of about 100-300 $\mathrm{nm}$. The high transmittance values in Figure 7 suggest that these aggregates have an open transparent structure. The ratio between the amplitudes of the slow and the fast relaxation modes is displayed in Figure 8. At low temperatures, the amplitudes are fairly independent of the temperature. The slow mode is more dominant for the samples with the higher amount of PropOx, indicating that these have a greater tendency to form aggregates due to the more hydrophobic nature. When the temperature approaches region ii, the slow mode is becoming more dominant (increasing values of $A_{\mathrm{s}} / A_{\mathrm{f}}$ ), suggesting that the number of unassociated polymer chains in the samples is reduced as aggregation is promoted at higher temperatures.
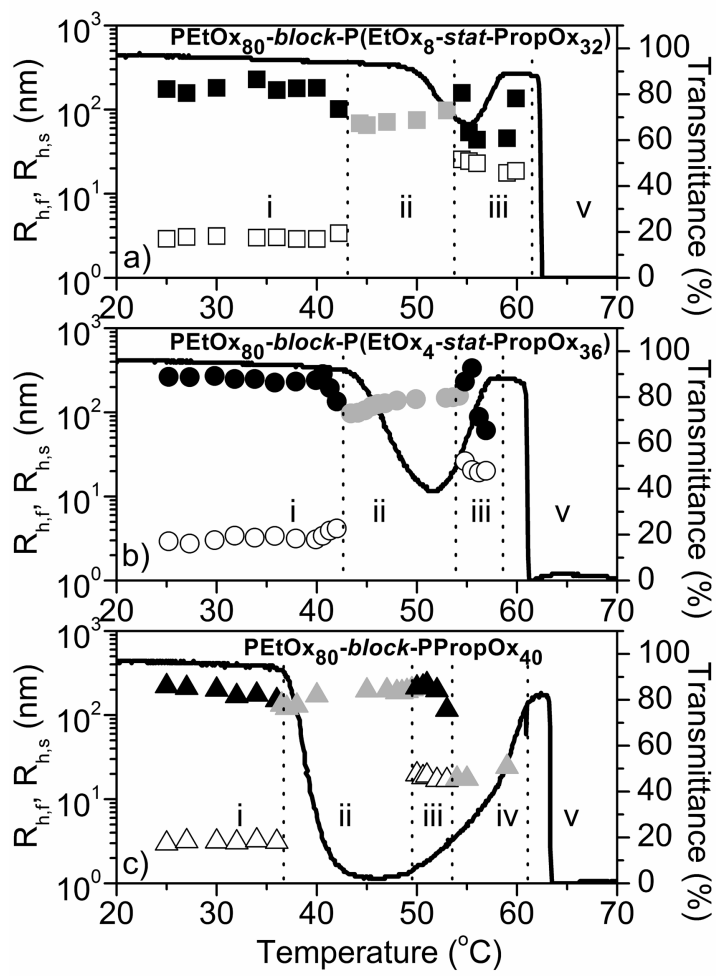

Figure 7. Apparent hydrodynamic radius of the indicated systems as a function of temperature at a polymer concentration of $5 \mathrm{mg} / \mathrm{mL}$. Open symbols represent sizes calculated from the fast relaxation mode, closed black symbols represent sizes calculated from the slow relaxation mode, and closed gray symbols represent sizes calculated from correlation functions that exhibit only one relaxation mode. The solid lines show the transmittance values for the same systems.

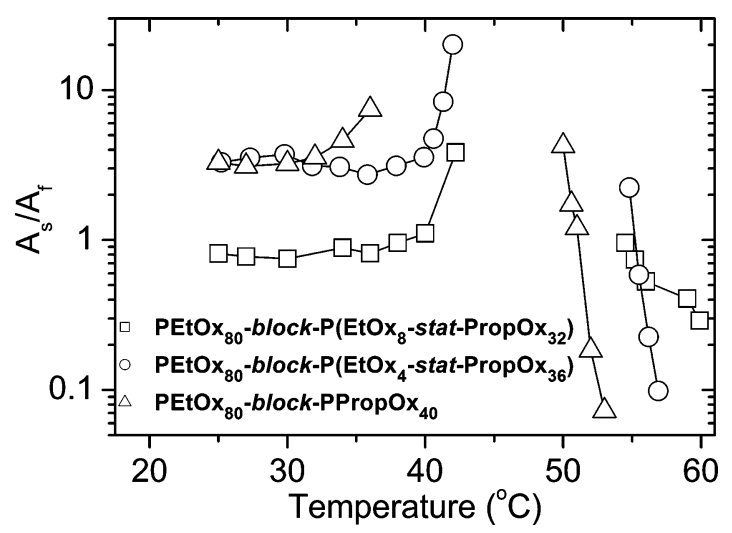

Figure 8. Ratio between the amplitude of the slow and the fast relaxation mode for the conditions where two relaxation modes are observed.

As mentioned above, a temperature increase will cause the polymers to become more hydrophobic, even below their LCST, which is why the transmittance data are gradually decreasing throughout this temperature region (see Figure 7). This enhanced hydrophobicity will promote both shrinking of the aggregates and further aggregation, both of which will cause a reduction in the transmittance data (Table 1). It is reasonable to assume that both processes are occurring simultaneously for each of the three polymers. The overall change in aggregate sizes displayed in Figure 7 (closed symbols) will then be the 
result of the combined effect of these two phenomena. As can be seen from Figure 6, the aggregates formed by $\mathrm{PEtO}_{80}-$ block$\mathrm{P}\left(\mathrm{EtOx}_{4}\right.$-stat-PropO $\left.\mathrm{x}_{36}\right)$ is somewhat larger than the aggregates formed by the two other samples. This is probably because this polymer has a higher tendency to aggregate than the more hydrophilic $\mathrm{PEtO}_{80}$-block-P(EtOx $\mathrm{x}_{8}$-stat-PropO $\left.\mathrm{x}_{32}\right)$, but it is not as prone to contraction as the more hydrophobic $\mathrm{PEtOx}_{80^{-}}$ block-PPropOx $\mathrm{x}_{40}$.

The stretched exponent, $\beta$, for the slow mode is displayed in Figure 9. This quantity is a measure of the width of the

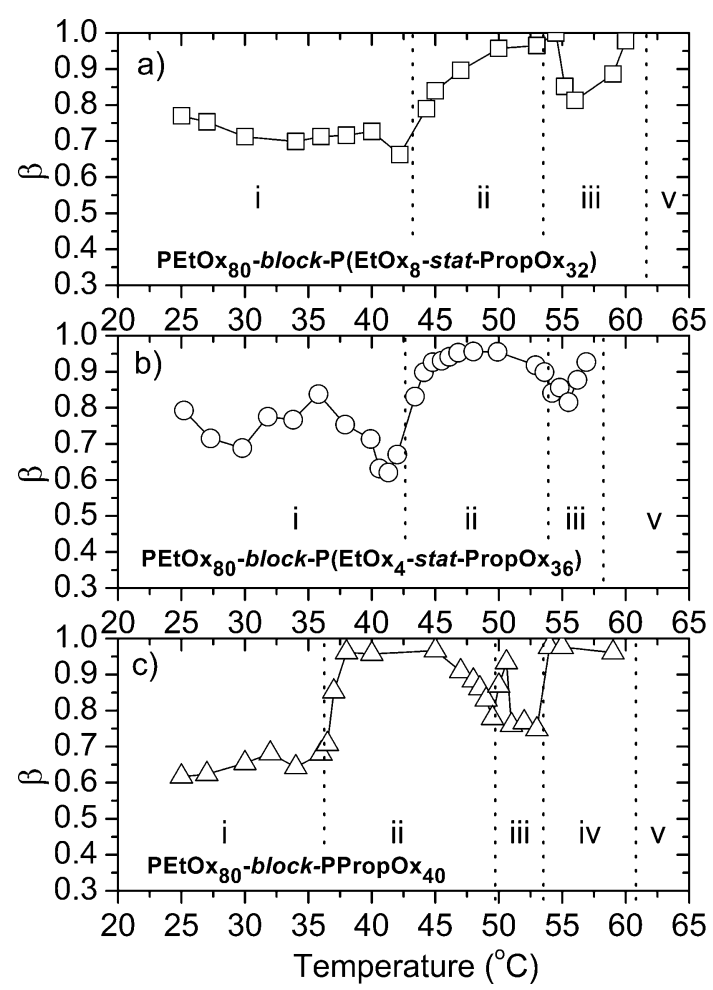

Figure 9. Stretched exponent $\beta$ (representing the width of the distribution of relaxation times) of the indicated systems at a polymer concentration of $5 \mathrm{mg} / \mathrm{mL}$.

distribution of relaxation times. Accordingly, it provides information about how broad the size distribution of the aggregates is. $\beta=1$ indicates a monodisperse distribution, and a broader size distribution will give lower values of $\beta$. The $\beta$ values in region $\mathrm{i}$ vary between 0.6 and 0.7 for $\mathrm{PEtOx}_{80}$-block$\mathrm{PPropOx}_{40}$ and between 0.6 and 0.8 for the two other polymers. This suggests that the aggregates formed in this temperature region have a fairly broad size distribution, especially for the polymer with the highest content of PropOx.

Region ii. When the temperature is increased further, only one mode is evident in the correlation functions. The disappearance of the unimers from the correlation functions is most likely caused by the increased hydrophobicity of the polymers at elevated temperatures. This gives rise to enhanced aggregation, leaving none or very few unassociated polymer chains in the solution. As discussed above, the gradual disappearance of the unimers is observed already at the end of region i. In region ii, the sizes of the aggregates are increasing slightly when the temperature is raised. At the same time, Figure 7 shows that there is a pronounced drop in the transmittance of the samples. The combined size increase and transmittance decrease show that the samples are aggregating
(Table 1). However, the moderate increase in sizes is not sufficient to cause the drastic fall in the transmittance data. It is therefore reasonable to assume that while the polymer aggregates are sticking together into larger aggregates, they are also further dehydrated, leading to contraction and thereby reducing the overall size increase and enhancing the drop in the transmittance data.

From Figure 6, it is evident that the sizes of the aggregates become larger as the amount of PropOx in the copolymers is increased, showing that the higher hydrophobicity of these samples promotes aggregation. As can be seen from Figure 4, the enhanced associative forces caused by a higher content of PropOx also give rise to a more pronounced drop in the transmittance data. This is because both aggregation and contraction of the polymer species are promoted by the higher hydrophobicity. In addition, the onset of the drop in transmittance data occur in the middle of region ii for $\mathrm{PEtO}_{80}$-block-P $\left(\mathrm{EtOx}_{8}\right.$-stat-PropO $\left.\mathrm{x}_{32}\right)$, while it coincides with the transition from region $\mathrm{i}$ to region ii for the polymers with a higher content of PropOx. The transition between region $i$ and region ii marks the point where the unimers are no longer detectable in the samples. This suggests that for the most hydrophobic copolymers with a high content of PropOx the enhanced hydrophobicity will also cause a pronounced aggregation and contraction of the aggregates at this point, causing the onset of the first cloud point. However, for the more hydrophilic $\mathrm{PEtO}_{80}$-block-P $\left(\mathrm{EtO}_{8}\right.$-stat-PropO $\left.\mathrm{x}_{32}\right)$, the higher content of EtOx in the second block delays the onset of the cloud point.

At the start of region ii, the $\beta$ values are increasing toward 1 (see Figure 9), indicating that the size distribution of the aggregates are becoming more monodisperse. The appearance of relatively monodisperse aggregates has also been observed for other thermoresponsive systems. ${ }^{15,48}$ A possible explanation for this phenomenon is that while enhanced hydrophobicity of the polymer will increase the sticking probability, the sticking probability of the particles is reduced when the volume fraction, $\varphi$, of polymer inside the aggregates and the aggregation number, $N_{\mathrm{agg}}$, is increased. ${ }^{32,54-56}$ If the balance between the hydrophobicity, $\varphi$ and $N_{\text {agg }}$ reaches a state where the sticking probability goes toward zero for a certain aggregation number, smaller particles will continue to aggregate until this aggregation number is reached. Particles with a higher aggregation number will have a very low sticking probability and mostly stay at their current size. As a result the overall size of the particles will go toward a constant value, above which aggregation is suppressed. Consequently the particle size distribution becomes practically monodisperse. Changing the hydrophobicity of the aggregates by varying the temperature will result in a shift in the optimum size, but the monodisperse nature of the aggregates will be retained. At the end of region ii, the $\beta$-values of $\mathrm{PEtOx}_{80}$-block-PPropOx $\mathrm{x}_{40}$ and $\mathrm{PEtO}_{80}$-block$\mathrm{P}\left(\mathrm{EtOx}_{4}\right.$-stat-PropO $\left.\mathrm{x}_{36}\right)$ are decreasing again. At this stage PropOx is becoming very hydrophobic, and as a result the stickiness of the aggregates increases significantly. The aggregation process is no longer limited by an optimal value of $N_{\mathrm{agg}}$, and the aggregates therefore become more polydisperse again. $\mathrm{PEtOx}_{80}$-block-P $\left(\mathrm{EtOx}_{8}\right.$-stat-PropOx $\left.\mathrm{x}_{32}\right)$ does not contain enough PropOx to render the aggregates sufficiently hydrophobic, and thus the $\beta$-values continues to increase throughout region ii (see Figure 9a). 
The transition from region i to region ii occurs earlier as the amount of PropOx in the copolymers is raised due to the higher hydrophobicity.

Region iii. At even higher temperatures, a second mode reappears in the correlation functions. However, the sizes of both modes are much larger than the unimers, suggesting that there is a coexistence of aggregates of two different sizes. One size is very close to the size observed at lower temperatures in region ii, while the other particles are about 10 times smaller. As can be seen from Figure 8, the ratio between the amplitude of the slow and the fast mode is decreasing as the temperature is raised. This indicates that the amount of small aggregates is increasing. Accordingly, the large particles are gradually changing into smaller particles. Such a size decrease can either be caused by contraction of the aggregates into smaller and more compact particles, or it can be due to a fragmentation of the aggregates into smaller entities. The sizes are decreased with a factor of $\sim 10$. This would indicate that if the particles were contracting they would become 1000 times more compact, which is rather unlikely. Also, in this temperature region, the transmittance of the sample is increasing (see Figure 7). Since contraction, resulting from dehydration, of the particles into such small sizes would cause a further decrease of the transmittance data (see Table 1), it is clear that the large aggregates are actually gradually fragmenting into smaller structures. Considering that the polymers are expected to become more hydrophobic as the temperature is raised, the fragmentation of the large aggregates is somewhat surprising. However, in this temperature region, the PropOx block is much more hydrophobic than the EtOx block. ${ }^{13}$ This will cause a gradual rearrangement of the polymer chains into structures where several PropOx blocks are clustering together, surrounded by EtOx blocks. Since the EtOx block is still quite hydrophilic in this temperature region, ${ }^{12,13}$ they will form a relatively hydrophilic shell around the hydrophobic PropOx core, leading to the formation of micelle-like structures with a much smaller size than the original clusters. The fast mode can be represented by a single exponential, illustrating that these micelle-like structures have a narrow size distribution.

As can be seen from Figure 7, the size of the micelle-like clusters (open symbols in region iii) is becoming smaller as the temperature is increased, suggesting that they are contracting. This is probably due to a dehydration of the EtOx block as it gradually becomes more hydrophobic at elevated temperatures. The temperature effect on the size of the larger aggregates is more complex and is probably caused by an interplay between aggregation, contraction, and possibly also fragmentation. This combination of different effects also causes the $\beta$-values in Figure 9 to vary throughout this region.

Since the transition from region ii to region iii is caused by the incipient fragmentation of the aggregates, it is expected to coincide with the minimum in the transmittance data. From Figure 7 it can be seen that the transmittance minimum for the two copolymers with the highest amounts of PropOx occur at somewhat lower temperatures than the transition from region ii to region iii. The reason for this is probably that when very few micelle-like structures are present in the samples, the scattering from the larger aggregates is dominating the correlation functions. Accordingly, only one relaxation mode is observed until the amount of micelle-like structures is high enough to be detected. Therefore, the transition from region ii to region iii occurs at higher temperatures than the minimum in the transmittance data. For $\mathrm{PEtO}_{80}-$ block-P $\left(\mathrm{EtOx}_{8}\right.$-stat-PropO $\left.\mathrm{x}_{32}\right)$ the transmittance minimum is located at a slightly higher temperature than the transition from region ii to region iii. As can be seen from Figure $7 \mathrm{a}$, the large aggregates are still growing at this stage. Therefore, in a limited temperature range the decreased transmittance due to the growth of the large aggregates dominates over the rise in transmittance caused by the fragmentation into smaller structures. The minimum in the transmittance data is shifted toward lower temperatures as the amount of PropOx is raised due to the higher hydrophobicity.

Region iv. For PEtOx $\mathrm{x}_{80}$-block-PPropOx $\mathrm{x}_{40}$ all the large aggregates are eventually fragmented into the smaller micellelike structures, and accordingly only one relaxation mode is then observed in the correlation functions. The $\beta$-values (Figure 9c) are very close to one, indicating that the micellelike structures have a narrow size distribution. The transmittance data are continuing to increase throughout this region, while the sizes observed by DLS are becoming slightly larger (Figure 7c). A combined size and transmittance increase indicates that the micelle-like structures are swelling (Table 1), possibly due to a rearrangement of the polymer entities causing the EtOx chains located at the surface to extend further out from the particles.

This stage (region iv) is not observed for $\mathrm{PEtOx}_{80}$-block$\mathrm{P}\left(\mathrm{EtOx}_{8}\right.$-stat-PropOx $\left.\mathrm{x}_{32}\right)$ and $\mathrm{PEtO}_{80}$-block-P(EtOx $\mathrm{x}_{4}$-stat-Pro$\left.\mathrm{pOx}_{36}\right)$, probably because the $\mathrm{P}(\mathrm{EtOx}$-stat-PropOx) block in some cases contains too much EtOx to form a sufficiently pure hydrophobic core to rearrange the copolymers into the micellelike structures.

Region v. At very high temperatures, there is an abrupt drop in the transmittance data. At this stage we observe multiple scattering in the DLS data, and accordingly the data cannot be analyzed. The drop in transmittance data and the multiple scattering suggest the formation of large and compact particles. At this stage EtOx is also becoming very hydrophobic due to dehydration, causing a pronounced association of the samples. Interestingly, as can be seen from Figure 4, this second cloud point is occurring at a higher temperature for $\mathrm{PEtO}_{80}{ }^{-}$ block-PPropO $\mathrm{x}_{40}$ than for the other two samples even though it is more hydrophobic. Since $\mathrm{PEtOx}_{80}$-block-PPropOx ${ }_{40}$ reaches a state where all of the large polymer aggregates are split into smaller micelle-like structures in region iv, the more hydrophilic shell of these structures probably suppresses further aggregation causing the cloud point to shift to a higher temperature.

Static Light Scattering. Since PEtOx ${ }_{80}$-block-PPropOx $\mathrm{x}_{40}$ has a region where all the larger aggregates are split into the micelle-like structures (region iv), it is also possible to examine the fragmentation process by SLS. For the other samples, the coexistence of micelle-like structures and larger aggregates would make the interpretation of the SLS data ambiguous, since the scattered intensities would be influenced by structures of two different sizes. We have therefore conducted SLS experiments on $\mathrm{PEtOx}_{80}$-block-PPropO $\mathrm{x}_{40}$ at $45{ }^{\circ} \mathrm{C}$, where the DLS data show that only the large aggregates are present, and at $55{ }^{\circ} \mathrm{C}$, where the DLS data indicate that the sample only contains the micelle-like structures. For comparison, the sample was also measured at $25{ }^{\circ} \mathrm{C}$. The results from the SLS experiments are displayed in Figure 10. The data are analyzed using eq 3 , where the intercept with the $y$-axis gives $1 / M_{\mathrm{w}}$. At $25^{\circ} \mathrm{C}$, the scattering from the sample is influenced by both the large aggregates $\left(R_{\mathrm{h}, \mathrm{s}}=221 \mathrm{~nm}\right)$ and the copolymer unimers $\left(R_{\mathrm{h}, \mathrm{f}}=3.0 \mathrm{~nm}\right)$. Accordingly, the results from the SLS measurements give sizes $\left(R_{\mathrm{g}} \approx 14 \mathrm{~nm}\right)$ and molecular weights $\left(M_{\mathrm{w}} \approx 1 \times 10^{4}\right)$ that have values in the between these two 


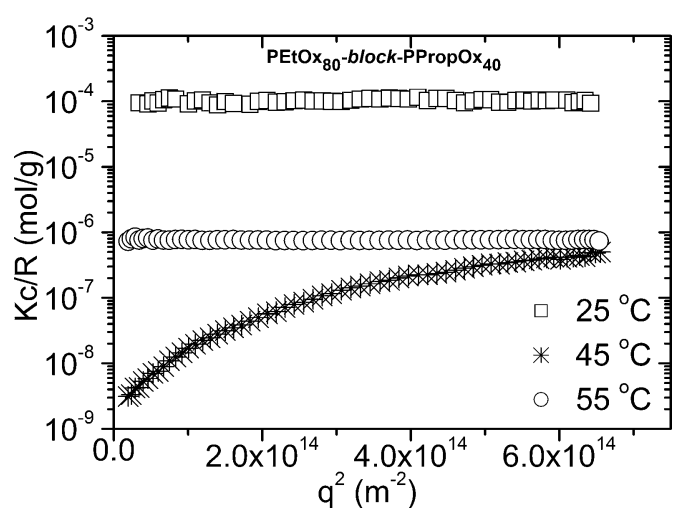

Figure 10. Static light scattering data for $\mathrm{PEtOx}_{80}$-block-PPropOx $\mathrm{x}_{40}$ at 25,45 , and $55{ }^{\circ} \mathrm{C}$. The $y$-axis has a logarithmic scale due to the large differences in the scattered intensities between the three temperatures.

structures. At $45^{\circ} \mathrm{C}$, large aggregates with $M_{\mathrm{w}} \approx 1 \times 10^{9}$ are formed. The huge structures formed at this temperature make it difficult to give a reasonable estimate of $R_{\mathrm{g}}$. When the temperature is raised to $55{ }^{\circ} \mathrm{C}$, the molecular weight of the associated structures is decreased to $M_{\mathrm{w}} \approx 1 \times 10^{6}$, confirming that the large structures formed at $45^{\circ} \mathrm{C}$ have fragmented into smaller structures. At $55{ }^{\circ} \mathrm{C}, R_{\mathrm{g}} \approx 15 \mathrm{~nm}$, and the ratio between the radius of gyration and the hydrodynamic radius is $R_{\mathrm{g}} / R_{\mathrm{h}} \approx$ 0.9. This is in good agreement with spherical micelles that usually have a value of $R_{\mathrm{g}} / R_{\mathrm{h}}$ in the range $0.8-1.0 .^{57}$

\section{CONCLUSIONS}

$\mathrm{PEtO}_{80}$-block-P(EtOx $\mathrm{x}_{x}$-stat-PropOx $\left.\mathrm{x}_{40-x}\right)$ block copolymers with $x=0,4$, or 8 reveal a complex aggregation behavior whereby the transmittance data go through a minimum before it falls off again at high temperatures. Figure 11 shows a

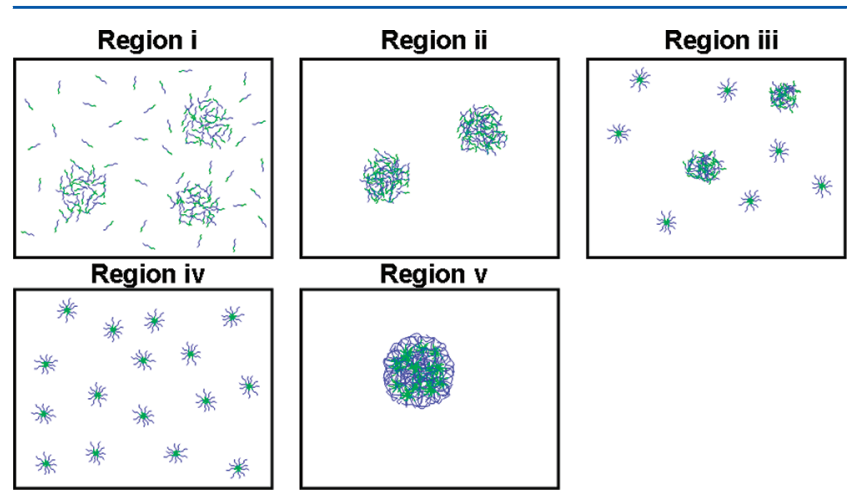

Figure 11. Schematic illustration of the different temperature regions observed, going from low temperatures in region $\mathrm{i}$ toward high temperatures in region $\mathrm{v}$.

schematic illustration of the different temperature regions observed for these copolymers. At low temperatures in region i, polymer unimers coexist with large polymer aggregates that have an open structure. This illustrates that the copolymers have a somewhat hydrophobic nature even at low temperatures. At higher temperatures (region ii), the hydrophobicity of the copolymers are increasing and practically all of the polymer units are in the form of aggregates. These aggregates are more compact than the aggregates observed in region $i$, and both continued aggregation and contraction of the aggregates are observed throughout region ii.
When the temperature is further increased to region iii, the aggregates are gradually fragmenting into micelle-like structures. This is due to a restructuring of the polymer aggregates into entities containing a core consisting mostly of PropOx (which is very hydrophobic in this temperature range) surrounded by a relatively hydrophilic EtOx shell. For $\mathrm{PEtOx}_{80}$-block-PPropOx $\mathrm{x}_{40}$ all the large aggregates are split into these micelle-like structures in region iv. For $\mathrm{PEtO}_{80^{-}}$ block-P(EtOx $x_{8}$ stat-PropOx $\left.\mathrm{x}_{32}\right)$ and $\mathrm{PEtOx}_{80}$-block-P(EtOx $\mathrm{E}_{4}$-stat$\mathrm{PropOx}_{36}$ ) such behavior is not observed. This is probably caused by a too high content of EtOx in the $\mathrm{P}(\mathrm{EtOx}$-statPropOx) block, preventing the formation of a core that is hydrophobic enough to form the micelle-like structures. Finally, at high temperatures in region $\mathrm{v}$, the enhanced hydrophobicity of the copolymers due to collapse of the EtOx block causes them to form large, compact aggregates.

\section{AUTHOR INFORMATION}

\section{Corresponding Author}

*E-mail: richard.hoogenboom@ugent.be (R.H.) or a.l. kjoniksen@kjemi.uio.no(A.-L.K.).

\section{Notes}

The authors declare no competing financial interest.

\section{REFERENCES}

(1) Wei, H.; Cheng, S. X.; Zhang, X. Z.; Zhuo, R. X. Prog. Polym. Sci. 2009, 34, 893-910.

(2) Liu, R.; Fraylich, M.; Saunders, B. R. Colloid Polym. Sci. 2009, 287, 627-643.

(3) Peng, C. L.; Tsai, H. M.; Yang, S. J.; Luo, T. Y.; Lin, C. F.; Lin, W. J.; Shieh, M. J. Nanotechnology 2011, 22, 265608.

(4) Vertommen, M. A. M. E.; Cornelissen, H.-J. L.; Dietz, C. H. J. T.; Hoogenboom, R.; Kemmere, M. F.; Keurentjes, J. T. F. J. Membr. Sci. 2008, 322, 243-248.

(5) Thorne, J. B.; Vine, G. J.; Snowden, M. J. Colloid Polym. Sci. 2011, 289, 625-646.

(6) Hu, J.; Liu, S. Macromolecules 2010, 43, 8315-8330.

(7) Pietsch, C.; Hoogenboom, R.; Schubert, U. S. Angew. Chem., Int. Ed. 2009, 48, 5653-5656.

(8) Pietsch, C.; Schubert, U. S.; Hoogenboom, R. Chem. Commun. 2011, 47, 8750-8765.

(9) Morris, G. E.; Vincent, B.; Snowden., M. J. J. Colloid Interface Sci. 1997, 190, 198-205.

(10) Chen, H.; Zhang, J.; Qian, Z.; Liu, F.; Chen, X.; Hu, Y.; Gu, Y. Nanotechnology 2008, 19, 185707.

(11) Idziak, I.; Avoce, D.; Lessard, D.; Gravel, D.; Zhu, X. X. Macromolecules 1999, 32, 1260-1263.

(12) Christova, D.; Velichkova, R.; Loos, W.; Goethals, E. J.; Du Prez, F. Polymer 2003, 44, 2255-2261.

(13) Hoogenboom, R.; Thijs, H. M. L.; Jochems, M. J. H. C.; van Lankvelt, B. M.; Fijten, M. W. M.; Schubert, U. S. Chem. Commun. 2008, 5758-5760.

(14) Xia, Y.; Burke, A. D.; Stöver, H. D. H. Macromolecules 2006, 39, $2275-2283$

(15) Kjøniksen, A. L.; Zhu, K.; Karlsson, G.; Nyström, B. Colloids Surf., A 2009, 333, 32-45.

(16) Ward, M. A.; Georgiou, T. K. Soft Matter 2012, 8, 2737-2745.

(17) Chee, C. K.; Hunt, B. J.; Rimmer, S.; Rutkaite, R.; Soutar, I.; Swanson, L. Soft Matter 2009, 5, 3701-3712.

(18) Carter, S.; Hunt, B.; Rimmer, S. Macromolecules 2005, 38, 4595-4603.

(19) Ward, M. A.; Georgiou, T. K. J. Polym. Sci., Polym. Chem. 2010, $48,775-783$.

(20) Siu, M.; He, C.; Wu, C. Macromolecules 2003, 36, 6588-6592.

(21) Wu, C.; Li, W.; Zhu, X. X. Macromolecules 2004, 37, 49894992. 
(22) Zhu, K.; Pamies, R.; Kjøniksen, A. L.; Nyström, B. Langmuir 2008, 24, 14227-14233.

(23) Hoogenboom, R.; Zorn, A.-M.; Keul, H.; Barner-Kowollik, C.; Moeller, M. Polym. Chem. 2012, 3, 335-342.

(24) Schild, H. G.; Muthukumar, M.; Tirrell, D. A. Macromolecules

1991, 24, 948-952.

(25) Hoogenboom, R.; Thijs, H. M. L.; Wouters, D.; Hoeppener, S.; Schubert, U. S. Soft Matter 2008, 4, 103-107.

(26) Eeckman, F.; Amighi, K.; Moës, A. J. Int. J. Pharm. 2001, 222, 259-270.

(27) Hua, F.; Jiang, X.; Zhao, B. Macromolecules 2006, 39, 34763479.

(28) Zhu, K.; Jin, H.; Kjøniksen, A. L.; Nyström, B. J. Phys. Chem. B 2007, 111, 10862-10870.

(29) Magnusson, J. P.; Khan, A.; Pasparakis, G.; Saeed, A. O.; Wang, W.; Alexander, C. J. Am. Chem. Soc. 2008, 130, 10852-10853.

(30) Weiss, J.; Böttcher, C.; Laschewsky, A. Soft Matter 2011, 7, 483-492.

(31) Lechner, M. D. J. Serb. Chem. Soc. 2005, 70, 361-369.

(32) Jonassen, H.; Kjøniksen, A. L. Phys. Rev. E 2011, 84, 022401.

(33) Hoogenboom, R. Angew. Chem., Int. Ed. 2009, 48, 7978-7994.

(34) Weber, C.; Hoogenboom, R.; Schubert, U. S. Prog. Polym. Sci.

2012, 37, 686-714.

(35) Park, J. S.; Katakoa, K. Macromolecules 2007, 40, 3599-3609.

(36) Huber, S.; Jordan, R. Colloid Polym. Sci. 2008, 286, 395-402.

(37) Luxenhofer, R.; Schulz, A.; Roques, C.; Li, S.; Bronich, T. K.; Batrakova, E. V.; Jordan, R.; Kabanov, A. V. Biomaterials 2010, 31, 4972-4979.

(38) von Erlach, T.; Zwicker, S.; Pidhatika, B.; Konradi, R.; Textor, M.; Hall, H.; Lühmann, T. Biomaterials 2011, 32, 5291-5303.

(39) Woodle, M. C.; Engbers, C. M.; Zalipsky, S. Bioconjugate Chem. 1994, 5, 493-496.

(40) Zalipsky, S.; Hansen, C. B.; Oaks, J. M.; Allen, T. M. J. Pharm. Sci. 1996, 85, 133-137.

(41) Rathna, G. V. N. J. Mater. Sci.: Mater. Med. 2008, 19, 23512358.

(42) Wiesbrock, F.; Hoogenboom, R.; Leenen, M.; van Nispen, S. F. G. M.; van der Loop, M.; Abeln, C. H.; van den Berg, A. M. J.; Schubert, U. S. Macromolecules 2005, 38, 7957-7966.

(43) Siegert, A. J. F. Massachusetts Institute of Technology: Radiation Laboratory Report No. 465, 1943.

(44) Ngai, K. L. Adv. Colloid Interface Sci. 1996, 64, 1-43.

(45) Tirado-Miranda, M.; Haro-Pérez, C.; Quesada-Pérez, M.; Callejas-Fernández, J.; Hidalgo-Álvarez, R. J. Colloid Interface Sci. 2003, 263, 74-79.

(46) Ellis, A. R.; Schaller, J. K.; McKiernan, M. L.; Selser, J. C. J. Chem. Phys. 1990, 92, 5731-5743.

(47) Zimm, B. H. J. Chem. Phys. 1948, 16, 1099-1116.

(48) Zhang, G.; Wu, C. Adv. Polym. Sci. 2006, 195, 101-176.

(49) Al-Manasir, N.; Zhu, K.; Kjøniksen, A. L.; Knudsen, K. D.; Karlsson, G.; Nyström, B. J. Phys. Chem. B 2009, 113, 11115-11123.

(50) Madsen, J.; Armes, S. P.; Bertal, K.; MacNeil, S.; Lewis, A. L. Biomacromolecules 2009, 10, 1875-1887.

(51) Zhao, J.; Zhang, G.; Pispas, S. J. Polym. Sci., Part A: Polym. Chem. 2010, 48, 2320-2328.

(52) Motokawa, R.; Morishita, K.; Koizumi, S.; Nakahira, T.; Annaka, M. Macromolecules 2005, 38, 5748-5760.

(53) Lutz, J. F.; Weichenhan, K.; Akdemir, Ö.; Hoth, A. Macromolecules 2007, 40, 2503-2508.

(54) Tanaka, H. Macromolecules 1992, 25, 6377-6380.

(55) Tanaka, H. Phys. Rev. Lett. 1993, 71, 3158-3161.

(56) Piçarra, S.; Martinho, J. M. G. Macromolecules 2001, 34, 53-58.

(57) Xie, D.; Xu, K.; Bai, R.; Zhang, G. J. Phys. Chem. B 2007, 111, $778-781$. 